\title{
Optimized Schwarz Methods with Robin Conditions for the Advection-Diffusion Equation
}

\author{
Olivier Dubois \\ McGill University, Department of Mathematics \& Statistics, 805 Sherbrooke, W \\ Montreal, Quebec, H3A 2K6, Canada. dubois@math.mcgill.ca \\ Summary. We study optimized Schwarz methods for the stationary advection- \\ diffusion equation in two dimensions. We look at simple Robin transmission con- \\ ditions, with one free parameter. In the nonoverlapping case, we solve exactly the \\ associated min-max problem to get a direct formula for the optimized parameter. \\ In the overlapping situation, we solve only an approximate min-max problem. The \\ asymptotic performance of the resulting methods, for small mesh sizes, is derived. \\ Numerical experiments illustrate the improved convergence compared to other Robin \\ conditions.
}

\section{Introduction}

The classical Schwarz method, first devised as a tool to prove existence and uniqueness results, converges only when there is overlap between subdomains, and converges very slowly for small overlap sizes. It was first proposed by Lions [Lio90] to change the Dirichlet transmission conditions in the algorithm to other types of conditions, in order to obtain a convergent nonoverlapping variant. More recently, optimized Schwarz methods were introduced by Japhet [Jap98]; using a Fourier analysis on a model problem, the convergence factor is uniformly minimized over a class of transmission conditions. The work of Japhet was originally carried on the advection-diffusion equation in the plane, without overlap, and using second order transmission conditions. Optimized Schwarz methods are now well-studied for symmetric partial differential equations, for example for the Laplace and modified Helmholtz equations (see [GHN01, Gan03] and references therein) and the Helmholtz equation (see [Gan01, GMN02]).

The purpose of this work is to study optimized Robin transmission conditions for the advection-diffusion equation, both in the case of nonoverlapping and overlapping domain decompositions. We start, in Section 2, by introducing the model problem in the plane. In Section 3, we present a general Schwarz iteration and its convergence factor, from which optimal transmission conditions can be found. We also briefly describe the Taylor polynomial approximations of the optimal symbols, a way to obtain local transmission operators. In Section 4 and 5, we present optimized Robin 
conditions, in the nonoverlapping and overlapping cases respectively. We illustrate our results in Section 6 with numerical experiments.

\section{The Model Problem}

The derivation and analysis of optimized Schwarz methods is done on a model problem. Here we consider the advection-diffusion equation on the plane with constant coefficients

$$
\left\{\begin{aligned}
\mathcal{L} u:=-\nu \Delta u+\mathbf{a} \cdot \nabla u+c u=f \text { in } \mathbb{R}^{2}, \\
u \text { is bounded at infinity, }
\end{aligned}\right.
$$

where $\nu, c>0$ and $\mathbf{a}=(a, b)$. For the convergence analysis of the algorithms presented subsequently, it will be sufficient to look at the homogeneous problem only, $f \equiv 0$, by linearity. We decompose the plane into two subdomains $\Omega_{1}$ and $\Omega_{2}$ with an overlap of width $L$

$$
\Omega_{1}:=\left(-\infty, \frac{L}{2}\right) \times \mathbb{R}, \quad \Omega_{2}:=\left(-\frac{L}{2}, \infty\right) \times \mathbb{R}
$$

and we denote by $u_{i}^{n}$ the approximate solution in subdomain $\Omega_{i}$, at iteration $n$.

Our analysis is based on the Fourier transform in the $y$ variable

$$
\mathcal{F}_{y}[u(x, y)]=\hat{u}(x, k):=\frac{1}{\sqrt{2 \pi}} \int_{-\infty}^{\infty} u(x, y) e^{-i y k} d y .
$$

In Fourier space, the homogeneous advection-diffusion equation becomes

$$
-\nu \frac{\partial^{2} \hat{u}}{\partial x^{2}}+a \frac{\partial \hat{u}}{\partial x}+\left(\nu k^{2}-i b k+c\right) \hat{u}=0 .
$$

This is a linear second order ODE in $x$ that can be solved analytically. The roots to the corresponding characteristic equation are given by

$$
\lambda^{ \pm}(k)=\frac{a \pm \sqrt{a^{2}+4 \nu c-4 i \nu b k+4 \nu^{2} k^{2}}}{2 \nu},
$$

where $\operatorname{Re}\left(\lambda^{+}\right)>0$ and $\operatorname{Re}\left(\lambda^{-}\right)<0$. The two fundamental solutions are then

$$
e^{\lambda^{+}(k) x}, \quad e^{\lambda^{-}(k) x} .
$$

We introduce the convenient notation

$$
\begin{aligned}
z(k) & :=\sqrt{a^{2}+4 \nu c-4 i \nu b k+4 \nu^{2} k^{2}}, \\
\xi(k) & :=\operatorname{Re}(z(k)), \quad \eta(k):=\operatorname{Im}(z(k)) .
\end{aligned}
$$




\section{Optimal Conditions and Taylor Approximations}

We first consider a general Schwarz iteration of the form

$$
\begin{aligned}
& \left\{\begin{aligned}
\mathcal{L} u_{1}^{n+1} & =0 & & \text { in }\left(-\infty, \frac{L}{2}\right) \times \mathbb{R}, \\
\frac{\partial u_{1}^{n+1}}{\partial x}-\mathcal{S}_{1}\left(u_{1}^{n+1}\right) & =\frac{\partial u_{2}^{n}}{\partial x}-\mathcal{S}_{1}\left(u_{2}^{n}\right) & & \text { at } x=\frac{L}{2},
\end{aligned}\right. \\
& \left\{\begin{aligned}
\mathcal{L} u_{2}^{n+1} & =0 & & \text { in }\left(-\frac{L}{2}, \infty\right) \times \mathbb{R}, \\
\frac{\partial u_{2}^{n+1}}{\partial x}-\mathcal{S}_{2}\left(u_{2}^{n+1}\right) & =\frac{\partial u_{1}^{n}}{\partial x}-\mathcal{S}_{2}\left(u_{1}^{n}\right) & & \text { at } x=-\frac{L}{2} .
\end{aligned}\right.
\end{aligned}
$$

where $\mathcal{S}_{i}$ are linear operators acting in the $y$ variable only, with Fourier symbols $\sigma_{i}$

$$
\mathcal{F}_{y}\left[\mathcal{S}_{i}(u)\right]=\sigma_{i}(k) \hat{u}(x, k) .
$$

Using the Fourier transform in $y$, we can solve each subproblem analytically, and find a convergence factor.

Proposition 1. The convergence factor of the Schwarz iteration (3)-(4) in Fourier space is

$$
\rho\left(k, L, \sigma_{1}, \sigma_{2}\right):=\left|\frac{\hat{u}_{1}^{n+1}\left(\frac{L}{2}, k\right)}{\hat{u}_{1}^{n-1}\left(\frac{L}{2}, k\right)}\right|=\left|\frac{\left(\lambda^{-}-\sigma_{1}\right)\left(\lambda^{+}-\sigma_{2}\right)}{\left(\lambda^{+}-\sigma_{1}\right)\left(\lambda^{-}-\sigma_{2}\right)} e^{-L\left(\lambda^{+}-\lambda^{-}\right)}\right|,
$$

where $\lambda^{ \pm}(k)$ are defined by $(1)$.

By choosing $\sigma_{1}(k)=\lambda^{-}(k)$ and $\sigma_{2}(k)=\lambda^{+}(k)$, we can make the convergence factor become 0 , and hence obtain an optimal convergence in 2 iterations only. This gives optimal operators $\mathcal{S}_{i}^{\text {opt }}$ when transforming back to real space, which turn out to be Dirichlet-to-Neumann maps, see for example [NR95]. However these operators are nonlocal in $y$ (their Fourier symbols $\lambda^{ \pm}$are not polynomials in $k$ ) and thus not convenient for practical implementation.

One way to find local conditions is to take, for $\sigma_{i}$, low order Taylor approximations of the optimal symbols $\lambda^{ \pm}$. For example, zeroth order approximations give

$$
\sigma_{1}=\frac{a-\sqrt{a^{2}+4 \nu c}}{2 \nu}, \quad \sigma_{2}=\frac{a+\sqrt{a^{2}+4 \nu c}}{2 \nu},
$$

which lead to a particular choice of Robin conditions. These methods work well only on small frequency components in $y$ (the Taylor approximations are good only for small $k$ ). An analysis of these methods can be found in [Jap97, Dub03].

\section{Optimized Robin Conditions Without Overlap}

We consider now a class of Robin transmission conditions by choosing

$$
\mathcal{S}_{1}(u)=\frac{a-p}{2 \nu} u, \quad \mathcal{S}_{2}(u)=\frac{a+p}{2 \nu} u,
$$

where $p$ is a real number. Using the general formula (5), the convergence factor for this choice reduces to 


$$
\rho_{R 1}(k, L, p):=\left|\frac{(p-z(k))^{2}}{(p+z(k))^{2}} e^{-\frac{L z(k)}{\nu}}\right|,
$$

where $z(k)$ is defined by (2). The idea of optimized Schwarz methods is, after fixing a class of conditions (Robin in this case), to minimize the convergence factor uniformly for all frequency components in a relevant range. This is formulated as a min-max problem. In our situation, a good value for the parameter $p$ is the one solving the optimization problem

$$
\min _{p \in \mathbb{R}}\left(\max _{k_{\min } \leq k \leq k_{\max }}\left|\rho_{R 1}(k, L, p)\right|\right) .
$$

In the following results, we use the short-hand notation $\xi_{\text {min }}:=\xi\left(k_{\text {min }}\right)$, $\xi_{\max }:=\xi\left(k_{\max }\right)$ and similar notations for $z_{\min }$ and $z_{\max }$.

Proposition 2 (Optimized Robin parameter, without overlap). If there is no overlap $(L=0)$, the unique minimizer $p^{*}$ of problem (8) is given by

$$
\begin{gathered}
p^{*}= \begin{cases}\left|z_{\min }\right| & \text { if } p_{c}<\left|z_{\min }\right|, \\
p_{c} & \text { if }\left|z_{\min }\right| \leq p_{c} \leq\left|z_{\max }\right|, \\
\left|z_{\max }\right| & \text { if } p_{c}>\left|z_{\max }\right|,\end{cases} \\
\text { where } p_{c}:=\sqrt{\frac{\xi_{\min }\left|z_{\max }\right|^{2}-\xi_{\max }\left|z_{\min }\right|^{2}}{\xi_{\max }-\xi_{\min }}} .
\end{gathered}
$$

For symmetric equations, the optimized Robin parameter is given by an equioscillation property, namely $\rho_{R 1}\left(k_{\text {min }}, 0, p^{*}\right)=\rho_{R 1}\left(k_{\max }, 0, p^{*}\right)$, see [Gan03]. On the other hand, for the advection-diffusion equation, this characterization does not always hold. Indeed, Proposition 2 shows that this equioscillation happens only when we fall in the middle case, i.e. when $p^{*}=p_{c}$.

Proposition 3 (Optimized Robin asymptotics, without overlap). For $L=0$ and $k_{\max }=\frac{\pi}{h}$, the asymptotic performance for small $h$ of the Schwarz method with optimized Robin transmission conditions is

$$
\max _{k_{\min } \leq k \leq \frac{\pi}{h}}\left|\rho_{R 1}\left(k, 0, p^{*}\right)\right|=1-2 \sqrt{\frac{2 \xi_{\min }}{\pi \nu}} h^{\frac{1}{2}}+O(h) .
$$

Note that the optimized Robin method has better asymptotic performance compared to the zeroth order Taylor approximation (6), which yields an expansion of the form $1-O(h)$ for small $h$. The proof of Proposition 2 and 3 can be found in [Dub03].

Remark 1. We can also choose two different constants in the Robin conditions

$$
\mathcal{S}_{1}(u)=\frac{a-p}{2 \nu} u, \quad \mathcal{S}_{2}(u)=\frac{a+q}{2 \nu} u,
$$

and look for a good pair of parameters $(p, q)$ by solving the min-max problem

$$
\min _{p, q \in \mathbb{R}}\left(\max _{k_{\min } \leq k \leq k_{\max }}\left|\frac{(p-z)(q-z)}{(p+z)(q+z)} e^{-\frac{L z}{\nu}}\right|\right) .
$$

This will be referred to as the optimized two-sided Robin conditions. In this paper, when using these conditions, the parameters are computed by solving the min-max problem numerically; there are no complete analytical results yet. 
Fig. 1 shows, on the left, a comparison of the convergence factors for different nonoverlapping Schwarz methods using Robin conditions.

\section{Optimized Robin Conditions With Overlap}

We now consider the overlapping situation. The convergence factor (7) can be written as

$$
\left|\rho_{R 1}(k, L, p)\right|=\frac{(p-\xi)^{2}+\eta^{2}}{(p+\xi)^{2}+\eta^{2}} e^{-\frac{L \xi}{\nu}} .
$$

Instead of finding the exact solution to the min-max problem, we derive in this section an approximate parameter that works well asymptotically for small $h$. We observe that $\eta$ remains bounded: $|\eta(k)| \leq|b|, \forall k$. Hence we have the upperbound

$$
\left|\rho_{R 1}(k, L, p)\right| \leq \frac{(p-\xi)^{2}+b^{2}}{(p+\xi)^{2}+b^{2}} e^{-\frac{L \xi}{\nu}}=: Q(\xi, p) .
$$

Instead of minimizing $\rho$, for simplicity we solve an approximate min-max problem using the upper bound

$$
\min _{p \in \mathbb{R}}\left(\max _{\xi_{\min } \leq \xi \leq \xi_{\max }} Q(\xi, p)\right) .
$$

We take $k_{\max }=\infty$ in this case to avoid extra complications. We expect that the parameter we obtain from this optimization will be close to the optimized parameter from (8), when $|b|$ and $L$ are small.

Proposition 4 (Approximate Robin parameter, with overlap). Let $L>0$ and $k_{\max }=\infty$. Define the critical value

$$
\xi_{2}(p):=\sqrt{\frac{2 \nu p-L b^{2}+L p^{2}+2 \sqrt{\nu^{2} p^{2}-2 \nu L p b^{2}-L^{2} b^{2} p^{2}}}{L}},
$$

and let $p_{\text {min }}:=\sqrt{\xi_{\text {min }}^{2}+b^{2}}$. If $\xi_{2}\left(p_{\min }\right)$ is complex, or if $\xi_{2}\left(p_{\min }\right)<\xi_{\text {min }}$, or if

$$
Q\left(\xi_{\text {min }}, p_{\text {min }}\right)>Q\left(\xi_{2}\left(p_{\text {min }}\right), p_{\text {min }}\right),
$$

then the unique minimizer $p^{*}$ of problem (9) is $p^{*}=p_{\min }$. Otherwise, the unique minimizer is given by the unique root $p^{*}$ (larger than $p_{\text {min }}$ ) of the equation

$$
Q\left(\xi_{\min }, p^{*}\right)=Q\left(\xi_{2}\left(p^{*}\right), p^{*}\right) .
$$

Proposition 5 (Approximate Robin asymptotics with overlap). For $L=h$ and $k_{\max }=\frac{\pi}{h}$, the asymptotic performance of the optimized Schwarz method, with the Robin parameter $p^{*}$ obtained through Proposition 4, is given by

$$
\max _{\xi_{\min } \leq \xi \leq \xi_{\max }}\left|\rho_{R 1}\left(k, h, p^{*}\right)\right|=1-4\left(\frac{\xi_{\text {min }}}{\nu}\right)^{\frac{1}{3}} h^{\frac{1}{3}}+O\left(h^{\frac{2}{3}}\right) .
$$


The proof of these results can also be found in [Dub03]. In the special case when $b=0$ (advection is normal to the interface), no approximation is made and our results above give the optimized Robin parameter. The asymptotic performance of the exact optimized Robin conditions (from solving (8)) is expected to be the same as (10) up to order $h^{1 / 3}$, with the same constant.

Fig. 1 shows on the right the convergence factors obtained for four different Robin transmission conditions, when overlap is used.
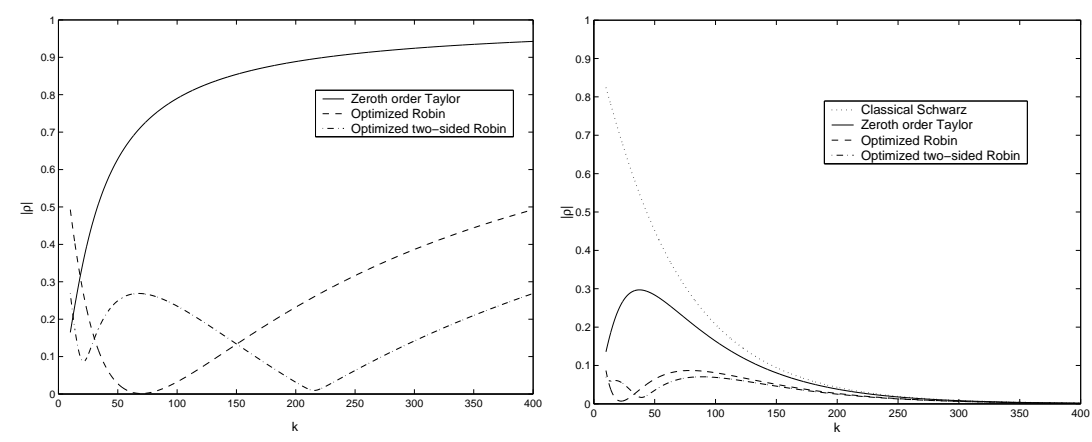

Fig. 1. Convergence factors for the values $\nu=0.1, a=1, b=1, c=1$, $\left[k_{\min }, k_{\max }\right]=[10,400]$. The case without overlap is shown on the left, and with overlap $L=\pi / 400$ on the right.

\section{Numerical Experiments}

We consider here an example with a varying advection $\mathbf{a}(x, y)$ obtained from a NavierStokes computation, see Fig. 2. The domain is the square $\Omega=(0, \pi)^{2}$, the viscosity is taken to be $\nu=0.1$, and $c=1$. The source term is given by $f(x, y)=\sin (5 x) \sin (5 y)$. The results were obtained using a finite difference solver, for rectangular domains. The original region is divided into two symmetric subdomains, with vertical interfaces. For the initial data to start the Schwarz iteration, we use vectors of random values, to make sure the initial error contains a wide

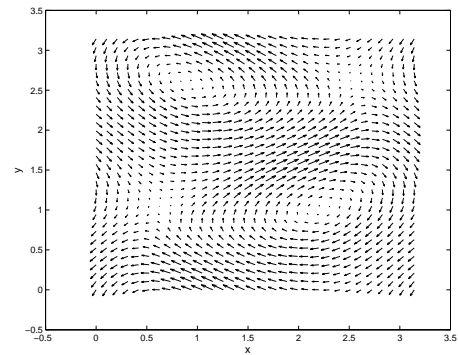

Fig. 2. The advection field. range of frequency components.

The optimized Schwarz methods are constructed using model problems with constant coefficients. When the coefficients are varying (continuously) in the domain, we need to find optimized conditions at each mesh point on the interfaces separately. In our setting the optimized Robin parameters will depend on $y$, i.e. $p^{*}=p^{*}(y)$. Note that the computation of the optimized conditions is done only once, before starting the Schwarz iteration. 
Fig. 3 shows the convergence of the different Schwarz methods, using both nonoverlapping and overlapping decompositions. Note that the effect of using an overlap is significant on the convergence: even by using a small overlap of only two grid spaces wide, the number of iterations required to reach tolerance is cut down by a factor of more than 2 .

We also looked at the effect of $h$ on the convergence rate of the Schwarz iteration. Fig. 4 shows logarithmic plots of the number of iterations needed to achieve an error of $10^{-6}$, for different values of the mesh size $h$. The numerical results agree well with the theoritical asymptotic performances: for those we have derived, but also those we expect for two-sided Robin conditions.
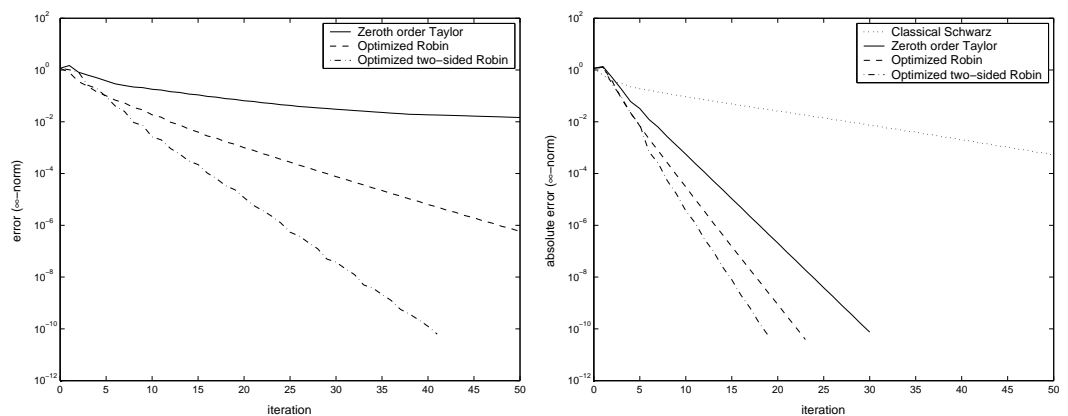

Fig. 3. Comparison of different transmission conditions for a varying advection, $\nu=0.1, c=1, h=\pi / 300$. The case without overlap is shown on the left, and the case with overlap $(L=2 h)$ on the right.
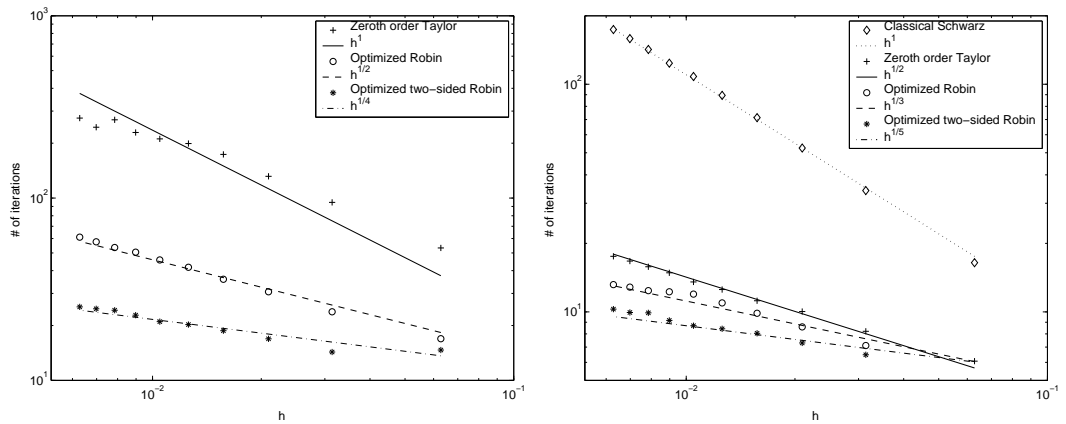

Fig. 4. Number of iterations needed to achieve an error of $10^{-6}$, for different values of $h$, without overlap on the left and with overlap $L=2 h$ on the right. 


\section{Conclusion}

We have computed optimized Robin transmission conditions in the Schwarz iteration for the advection-diffusion equation, by solving analytically the min-max problem. When the subdomains are not overlapping, the optimized parameter is given by an explicit formula. In the overlapping case, we have solved an approximate min-max problem only: computing the optimized parameter reduces to solving a nonlinear equation (in the worst case). The approximation we have made is good when the advection is not too strongly tangential to the interfaces, and for small mesh sizes $h$. The asymptotic performance of these optimized methods exhibits a weaker dependence on the mesh size than previously known Robin conditions.

\section{References}

[Dub03] Olivier Dubois. Optimized Schwarz methods for the advection-diffusion equation. Master's thesis, McGill University, 2003.

[Gan01] Martin J. Gander. Optimized Schwarz methods for Helmholtz problems. In Thirteenth international conference on domain decomposition, pages 245-252, 2001.

[Gan03] Martin J. Gander. Optimized Schwarz methods. Technical Report 2003-01, Dept. of Mathematics and Statistics, McGill University, 2003. In revision for SINUM.

[GHN01] Martin J. Gander, Laurence Halpern, and Frédéric Nataf. Optimized Schwarz methods. In Tony Chan, Takashi Kako, Hideo Kawarada, and Olivier Pironneau, editors, Twelfth International Conference on Domain Decomposition Methods, Chiba, Japan, pages 15-28, Bergen, 2001. Domain Decomposition Press.

[GMN02] Martin J. Gander, Frédéric Magoulès, and Frédéric Nataf. Optimized Schwarz methods without overlap for the Helmholtz equation. SIAM J. Sci. Comput., 24(1):38-60, 2002.

[Jap97] Caroline Japhet. Conditions aux limites artificielles et décomposition de domaine: Méthode oo2 (optimisé d'ordre 2). application à la résolution de problèmes en mécanique des fluides. Technical Report 373, CMAP (Ecole Polytechnique), 1997.

[Jap98] Caroline Japhet. Optimized Krylov-Ventcell method. Application to convection-diffusion problems. In Petter E. Bjørstad, Magne S. Espedal, and David E. Keyes, editors, Proceedings of the 9th international conference on domain decomposition methods, pages 382-389. ddm.org, 1998.

[Lio90] Pierre-Louis Lions. On the Schwarz alternating method. III: a variant for nonoverlapping subdomains. In Tony F. Chan, Roland Glowinski, Jacques Périaux, and Olof Widlund, editors, Third International Symposium on Domain Decomposition Methods for Partial Differential Equations, held in Houston, Texas, March 20-22, 1989, Philadelphia, PA, 1990. SIAM.

[NR95] Fréderíc Nataf and Francois Rogier. Factorization of the convectiondiffusion operator and the Schwarz algorithm. $M^{3} A S, 5(1): 67-93,1995$. 